\title{
Kaon differential flow in relativistic heavy-ion collisions
}

\author{
Bao-An Li, ${ }^{1, *}$ Bin Zhang, ${ }^{2, \dagger}$ Andrew T. Sustich, ${ }^{1, \$}$ and C. M. Ko ${ }^{2, \S}$ \\ ${ }^{1}$ Department of Chemistry and Physics, Arkansas State University, P.O. Box 419, State University, Arkansas 72467-0419 \\ ${ }^{2}$ Cyclotron Institute and Physics Department, Texas A\&M University, College Station, Texas 77843
}

(Received 29 January 1999; published 10 August 1999)

\begin{abstract}
Using a relativistic transport model, we study the azimuthal momentum asymmetry of kaons with fixed transverse momentum, i.e., the differential flow, in heavy-ion collisions at beam momentum of $6 \mathrm{GeV} / c$ per nucleon, available from the Alternating Gradient Synchrotron (AGS) at the Brookhaven National Laboratory (BNL). We find that in the absence of kaon potential the kaon differential flow is positive and increases with transverse momentum as that of nucleons. The repulsive kaon potential as predicted by theoretical models, however, reduces the kaon differential flow, changing it to negative for kaons with low transverse momenta. Cancellation between the negative differential flow at low momenta and the positive one at high momenta is then responsible for the experimentally observed nearly vanishing in-plane transverse flow of kaons in heavyion experiments. [S0556-2813(99)02109-3]
\end{abstract}

PACS number(s): 25.75.Ld, 13.75.Jz, 21.65.+f

Since the work of Kaplan and Nelson [1] on the possibility of a kaon condensation in the core of neutron stars, there have been many theoretical studies of kaon properties in dense matter [2]. It is now generally agreed that a kaon has a weak repulsive potential in nuclear matter while an antikaon has instead a strong attractive one. The latter is responsible for the existence of the kaon condensation that was originally proposed in Ref. [1]. Brown and Bethe [3] have further argued that because of the softening of the nuclear equation of state due to the kaon condensation, the maximum mass of neutron stars would decrease, hence allowing for the existence of mini black holes.

Since a hot dense matter can be created in the initial stage of high energy heavy ion collisions, a unique opportunity thus exists for studying the kaon in-medium properties [4-7]. Indeed, Li et al. [8] have shown that it is possible to extract the information on kaon potential or dispersion relation in dense matter from the kaon collective flow in heavy ion collisions. Specifically, they have found, based on the relativistic transport model, that the repulsive kaon potential in nuclear medium would reduce its flow relative to that of nucleons, while the flow of antikaons would be similar to that of nucleons due to their attractive potential [9]. Since then, many theoretical studies [10-14] have been carried out, and all have reached the same conclusion. These theoretical studies have stimulated a number of experiments at both GSI and AGS. All experiments have shown that kaons have very small, if not zero, flow, i.e., both the kaon average in-plane transverse momentum as a function of rapidity and its slope at midrapidity are consistent with zero within the experimental error bars [15]. For example, both the FOPI and KAOS Collaborations at SIS/GSI [16-18] have found a negligible flow for $K^{+}$in collisions of $\mathrm{Ni}+\mathrm{Ni}$ at $E_{\text {beam }} / A=1.93 \mathrm{GeV}$ and $\mathrm{Au}+\mathrm{Au}$ at $1 \mathrm{GeV}$, respectively. Several collaborations at

\footnotetext{
*Electronic address: Bali@navajo.astate.edu

${ }^{\dagger}$ Electronic address: Bzhang@kopc1.tamu.edu

\#Electronic address: Sustich@navajo.astate.edu

§Electronic address: Ko@cycomp.tamu.edu
}

the AGS/BNL have also carried out the flow analysis for $K^{+}, K^{-}$, and $K_{s}^{0}$ in $\mathrm{Au}+\mathrm{Au}$ collisions at beam energies from 2 to $12 \mathrm{GeV} /$ nucleon, and no statistically significant kaon transverse flow has been seen either [19-22].

There are suggestions that the observed vanishing kaon flow may be due to the fact that kaons are produced isotropically from hadron-hadron collisions [20] or these colliding hadrons have opposite transverse flows, leading thus to a reduced collective flow of produced kaons [23]. However, all transport mode calculations $[8,10-14]$ have shown that the initial transverse flow of produced kaons is positive, similar to that of nucleons, and the resulting vanishing kaon flow is due to the repulsive kaon potential as first demonstrated by Li et al. [8].

Vanishing transverse flow has also been seen for nucleons in heavy-ion collisions at an incident energy called the balance energy. Using the differential flow analysis, which measures the azimuthal momentum asymmetry at fixed transverse momentum, two of the present authors [24] have shown that the zero transverse flow is due to the cancellation between the positive flow of nucleons with high transverse momentum and the negative flow of those with low transverse momentum. The latter results from the effect of an attractive nuclear mean-field potential. In this paper, we carry out a similar differential flow analysis for kaons in order to better understand the physics underlying the observed vanishing kaon flow. We shall show that the repulsive kaon potential in nuclear medium can indeed reduce the primordial positive in-plane transverse flow of kaons to zero or even negative values, leading to a strong differential flow, i.e., kaons with high and low transverse momenta flow in opposite directions. A vanishing total in-plane traverse flow is, nevertheless, obtained when the kaon differential flow is integrated over the transverse momentum.

Our study is based on a relativistic transport model (ART1.0) for heavy-ion collisions at AGS energies [25]. In this model the imaginary part of kaon self-energy is approximately treated by scatterings of the kaon with other hadrons, and its real part is given by the mean-field potential. We shall concentrate only on the collective flow of $K^{0}$, so the 
Coulomb potential does not need to be considered. Various approaches have been used to evaluate the kaon dispersion relation in dense matter [1,2]. As an illustration, we shall use in the present study the $K^{+}$dispersion relation that is determined from the kaon-nucleon scattering length $a_{K N}$ using the impulse approximation, i.e.,

$$
\omega\left(p, \rho_{b}\right)=\left[m_{K}^{2}+p^{2}-4 \pi\left(1+\frac{m_{K}}{m_{N}}\right) a_{K N} \rho_{b}\right]^{1 / 2},
$$

where $m_{K}$ and $m_{N}$ are the kaon and nucleon masses, respectively; $\rho_{b}$ is the baryon density and $a_{K N} \approx-0.255 \mathrm{fm}$ is the isospin-averaged kaon-nucleon scattering length. The $K^{+}\left(K^{0}\right)$ potential in nuclear medium can then be defined as

$$
U\left(p, \rho_{b}\right)=\omega\left(p, \rho_{b}\right)-\left(m_{K}^{2}+p^{2}\right)^{1 / 2},
$$

which gives a repulsive potential of about $30 \mathrm{MeV}$ for a kaon at rest in normal nuclear matter.

The transverse collective flow has usually been studied by analyzing the average transverse momentum per particle in the reaction plane as a function of rapidity $y$ [27], i.e.,

$$
\begin{aligned}
\left\langle p_{x} / A\right\rangle(y) & =\frac{1}{A(y)} \sum_{i=1}^{A(y)} p_{i x} \\
& =\frac{1}{A(y)} \int p_{t} \frac{d N}{d p_{t}}\langle\cos \phi\rangle\left(y, p_{t}\right) d p_{t},
\end{aligned}
$$

where $A(y)$ is the number of particles at rapidity $y, \quad \phi$ is the azimuthal angle with respect to the reaction plane, and

$$
\langle\cos \phi\rangle\left(y, p_{t}\right) \equiv\left(\frac{d N}{d p_{t}}\right)^{-1} \int \cos \phi \frac{d^{2} N}{d p_{t} d \phi} d \phi .
$$

A nonvanishing $\langle\cos \phi\rangle\left(y, p_{t}\right)$ indicates the existence of an azimuthally asymmetric transverse flow at the rapidity $y$ and transverse momentum $p_{t}$. It is related to the first Fourier coefficient in the expansion $[28,29]$,

$$
\frac{d^{2} N}{d p_{t} d \phi}=\frac{d N}{d p_{t}}\left[1+\sum_{i=1}^{\infty} 2 \mathrm{v}_{i}\left(y, p_{t}\right) \cos (i \phi)\right]
$$

i.e., $\langle\cos \phi\rangle\left(y, p_{t}\right)=\mathrm{v}_{1}\left(y, p_{t}\right)$. In Ref. [24], the $p_{t}$ dependence of $\langle\cos \phi\rangle$ has been named the differential flow. It has been found that the differential analysis of nucleon flow at the balance energies is useful both for understanding the disappearance of nuclear collective flow and for extracting the information on the nuclear equation of state [24].

The transverse flow of baryons in heavy-ion collisions is an experimentally well established fact at all energies. At beam momentum $6 \mathrm{GeV} / c$, about half of the kaons are produced from collisions between baryons, one thus expects that they would obtain some collectivity as a result of the baryon collective flow. Meson-baryon collisions almost account for the other half. Since pions have a small directed flow, the directed flow of these kaons is thus weaker than those from baryon-baryon collisions. Overall, kaons are expected to have a weak, positive flow with respect to nucleons without

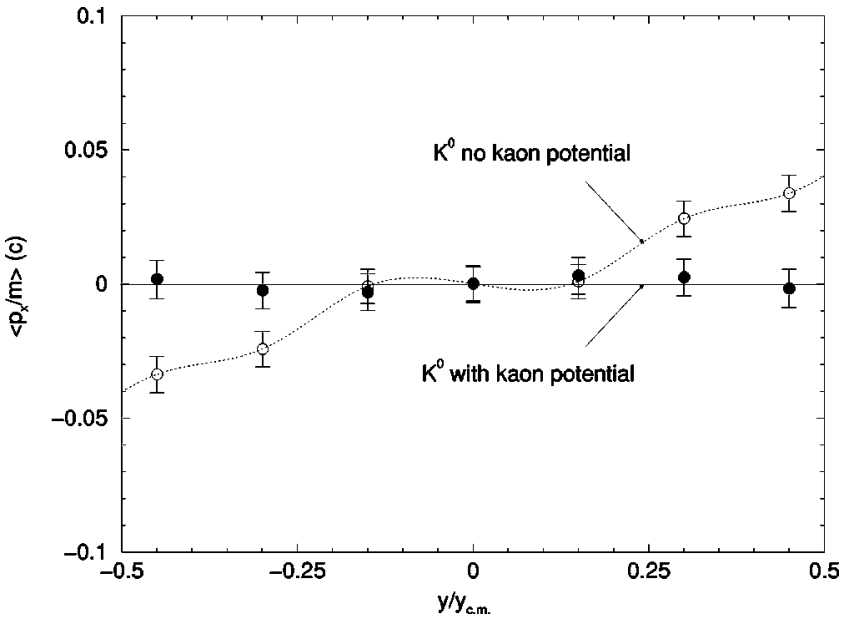

FIG. 1. The average transverse momentum of $K^{0}$ in the reaction plane for $\mathrm{Au}+\mathrm{Au}$ reactions at $P_{\text {beam }} / A=6 \mathrm{GeV} / c$ and an impact parameter of $4 \mathrm{fm}$. The open (filled) circles are the results obtained without (with) the kaon mean-field potential.

considering effects of the kaon potential. This is indeed seen in our calculations, e.g., for a soft equation of state without using the kaon potential, the average $p_{x} / m$ of those protons at $y / y_{\text {c.m. }}=0.5$ is around $0.1 c$ and is about twice as large as that of kaons. Because of the large anisotropy of the baryon distribution, especially in the later stage of the reaction, due to the baryon transverse flow, a repulsive kaon potential would then cause kaons to acquire a distribution that further differs from that of baryons. To demonstrate the effect of kaon potential, we compare results obtained with and without the kaon potential.

In Fig. 1, the average kaon transverse momentum (scaled by the kaon mass) in the reaction plane is shown as a function of rapidity (scaled by the beam rapidity) for $\mathrm{Au}+\mathrm{Au}$ collisions at an impact parameter of $4 \mathrm{fm}$ and beam momentum of $6 \mathrm{GeV} / c$ per nucleon. Experimental data from this reaction are being analyzed by both the E917 [20] and E895 [26] Collaborations. The open (filled) circles are the results obtained without (with) the kaon mean-field potential. As one expects, in the absence of potential kaons flow in the same direction as nucleons. However, there is essentially no flow in the whole rapidity range when the kaon mean-field potential is included in the transport model. This observation is in agreement with earlier findings at both lower [8] and higher energies [10], i.e., the kaon potential reduces its flow or even changes the flow direction. This strong dependence of the kaon flow on its potential in nuclear matter makes the kaon flow analysis a valuable tool to study the kaon inmedium properties.

To examine more closely the origin of vanishing kaon flow in the presence of a repulsive potential, we have carried out a differential flow analysis for kaons. Since we are considering collisions of symmetric nuclei, we include all kaons in the rapidity range of $\operatorname{abs}\left(y / y_{\text {c.m. }}\right) \leqslant 0.5$ by flipping the sign of $\langle\cos \phi\rangle$ for $y \leqslant 0$ in Eq. (4), so an improved statistic is obtained in evaluating the differential flow. The results are shown in Fig. 2, and it is seen that irrespective of the kaon transverse momentum its differential flow is always positive 


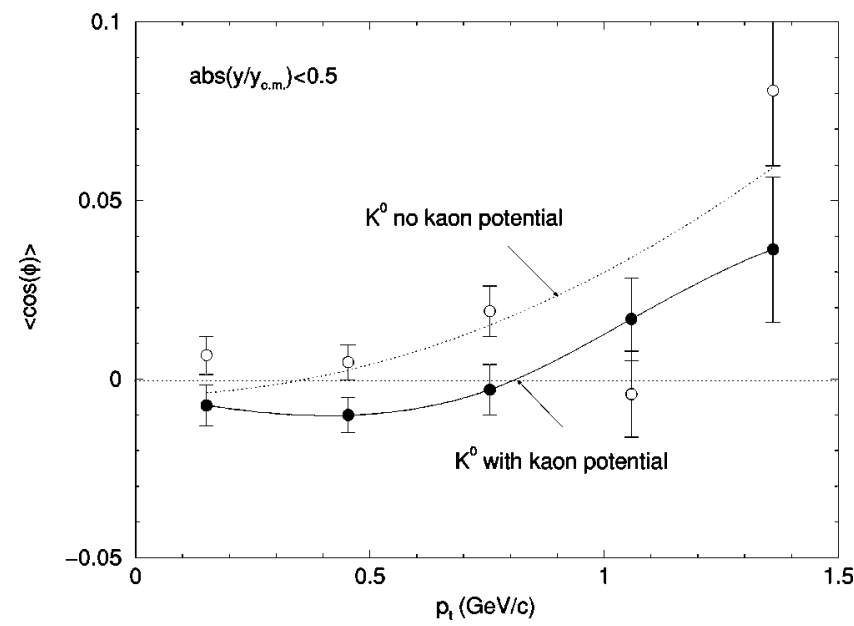

FIG. 2. The $K^{0}$ azimuthal asymmetry as a function of transverse momentum for the same reaction as in Fig. 1. The open (filled) circles are the results obtained without (with) the kaon mean-field potential.

when no potential is included. The value at $p_{t}$ around $1 \mathrm{GeV} / c$ is significantly below those at other transverse momenta, and this is related to the fact that these kaons are mainly produced in the space-time region where spectator matters present strong shadowing effects. With the repulsive kaon potential given by Eq. (2), the differential flow of kaons with transverse momenta less than about $0.8 \mathrm{GeV} / c$ becomes negative while that of kaons with higher transverse momenta remains positive. This change in the dependence of kaon differential flow on the transverse momentum follows from the fact that the force acting on a kaon is inversely proportional to its energy, so low energy kaons are more strongly repelled by baryons than high energy ones. The latter thus remain to flow in the same direction as baryons. Integrating over the transverse momentum distribution leads to a cancellation between opposite flows at low and high transverse momenta. The resulting kaon in-plane flow is therefore small as shown in Fig. 1.

The differential kaon flow has recently been studied by the E877 Collaboration [21,22], and it has indeed been found that there is a negative (positive) flow for $K^{+}$with low (high) transverse momenta. A similar analysis for kaon differential flow in the SIS/GSI experiments would be very useful in understanding the observed vanishing kaon flow.

In summary, using the relativistic transport model (ART) for heavy-ion collisions, we have studied the differential flow of kaons. We have found that the experimentally observed nearly zero in-plane transverse flow of kaons produced in heavy-ion collisions is due to the cancellation between their negative and positive differential flows at low and high transverse momenta. While the positive differential flow reflects the strong positive baryonic flow, the negative differential flow is caused by a repulsive in-medium kaon potential. The differential flow analysis thus provides a more detailed picture of kaon flow than the standard transverse flow analysis, and is therefore very useful for probing the in-medium kaon properties in heavy ion collisions.

This work was supported in part by NSF Grant No. PHY9870038, the Robert A Welch Foundation under Grant No. A-1358, and the Texas Advanced Research Program.
[1] D. B. Kaplan and A. E. Nelson, Phys. Lett. B 175, 57 (1986); 192, 193 (1987).

[2] G. E. Brown, K. Kubodera, and M. Rho, Phys. Lett. B 193, 273 (1987); H. D. Politzer and M. B. Wise, ibid. 273, 156 (1991); T. Muto and T. Tatsumi, ibid. 283, 165 (1992); G. E. Brown, C. M. Ko, and K. Kubodera, Z. Phys. A 341, 301 (1992); M. Lutz, A. Steiner, and W. Weise, Phys. Lett. B 278, 29 (1992); H. Yabu, S. Nakamura, F. Myhrer, and K. Kubodera, ibid. 315, 17 (1983); J. Schaffner-Bielich, A. Gal, I. N. Mishustin, H. Stöcker, and W. Greiner, ibid. 344, 268 (1994); G. E. Brown, C. H. Lee, M. Rho, and V. Thorsson, Nucl. Phys. A567, 937 (1994).

[3] G. E. Brown and H. Bethe, Astrophys. J. 423, 659 (1994).

[4] C. M. Ko, Z. G. Wu, L. H. Xia, and G. E. Brown, Phys. Rev. Lett. 66, 2577 (1991); Phys. Rev. C 43, 1881 (1991); X. S. Fang, C. M. Ko, G. E. Brown, and V. Koch, ibid. 47, 1678 (1993).

[5] X. S. Fang, C. M. Ko, G. Q. Li, and Y. M. Zheng, Phys. Rev. C 49, R608 (1994); Nucl. Phys. A575, 766 (1994).

[6] G. Q. Li, C. M. Ko, and X. S. Fang, Phys. Lett. B 329, 149 (1994).

[7] C. M. Ko and G. Q. Li, J. Phys. G 22, 1673 (1996); C. M. Ko, V. Koch, and G. Q. Li, Annu. Rev. Nucl. Part. Sci. 47, 505 (1997).
[8] G. Q. Li, C. M. Ko, and B. A. Li, Phys. Rev. Lett. 74, 235 (1995); G. Q. Li and C. M. Ko, Nucl. Phys. A594, 460 (1995); G. Q. Li, C. M. Ko, and G. E. Brown, Phys. Lett. B 381, 17 (1996).

[9] G. Q. Li and C. M. Ko, Phys. Rev. C 54, R2159 (1996).

[10] B. A. Li and C. M. Ko, Phys. Rev. C 54, 3283 (1996).

[11] Z. S. Wang et al., Nucl. Phys. A628, 151 (1997).

[12] E. L. Bratkovskaya, W. Cassing, and U. Mosel, Nucl. Phys. A622, 593 (1997).

[13] C. Fuchs, D. S. Kosov, A. Faessler, Z. S. Wang, and T. Waindzoch, Phys. Lett. B 434, 245 (1998).

[14] G. Q. Li and G. E. Brown, Nucl. Phys. A636, 487 (1998).

[15] W. Reisdorf, Nucl. Phys. A630, 15c (1998).

[16] FOPI Collaboration, J. L. Ritman et al., Z. Phys. A 352, 355 (1995).

[17] FOPI Collaboration, D. Best et al., Nucl. Phys. A625, 307 (1997).

[18] EOS Collaboration, Y. Shin et al., Phys. Rev. Lett. 81, 1576 (1998).

[19] C. A. Ogilvie for the E866 Collaboration, Advance in Nuclear Dynamics, edited by W. Bauer and A. Mignerey (Plenum, New York, 1997), p. 247.

[20] C. A. Ogilvie for the E802 and E917 Collaborations, Nucl. Phys. A629, 571c (1998). 
[21] M. Pollack, Ph.D. thesis, SUNY at Stony Brook, 1997.

[22] S. A. Voloshin for the E877 Collaboration, Nucl. Phys. A638, 455c (1998).

[23] C. David, C. Hartnack, and J. Aichelin, Nucl. Phys. A (to be published).

[24] B. A. Li and A. T. Sustich, Phys. Rev. Lett. 82, 5004 (1999).

[25] B. A. Li and C. M. Ko, Phys. Rev. C 52, 2037 (1995); 53, R22 (1996); Nucl. Phys. A601, 457 (1996).

[26] G. Rai et al. for the E895 Collaboration, talk given at
RHIC Winter Workshop, 1999, Lawrence Berkeley National Laboratory, URL http://www-rnc.lbl.gov/ nxu/workshop/ rww99program.html; E895 Collaboration, P. Chung et al., J. Phys. G 25, 255 (1999).

[27] P. Danielewicz and G. Odyniec, Phys. Lett. 157B, 146 (1985).

[28] S. A. Voloshin, Phys. Rev. C 55, R1630 (1997).

[29] A. M. Poskanzer and S. A. Voloshin, Phys. Rev. C 58, 1671 (1998). 